Lech Wołowski ${ }^{1}$

0000-0003-2012-1267

Uniwersytet Papieski Jana Pawła II w Krakowie

\title{
L'approccio agatologico-cristologico al concetto di persona nella riflessione teatrologica-teologica di Hans Urs von Balthasar e di Józef Tischner
}

Nel presente articolo ci occupiamo del problema della comprensione e della descrizione del concetto di persona nella riflessione teatrologica-teologica (teodrammatica) di due grandi pensatori del XX secolo, cioè di Hans Urs von Balthasar e di Józef Tischner. La scelta degli autori indica chiaramente che ci concentriamo sull'approccio moderno, contemporaneo, alla nozione di persona ed, in particolare, sull'approccio agatologico-cristologico, visto dalla prospettiva drammatica che caratterizza il pensiero filosofico-teologico di entrambi gli autori.

L'approccio drammatico determina, inoltre, abbastanza chiaramente le fonti sulle quali fondiamo la ricerca. Nel caso di Balthasar,

1 Dr. Lech Wołowski, Istituto di Dogmatica della Facoltà di Teologia dell'Università Giovanni Paolo II di Cracovia, dottorato: La grazia e la libertà nel dramma dell'uomo bisognoso della salvezza in H. U. von Balthasar e J. Tischner, Università Gregoriana, Roma 2017. Campi di ricerca: teodrammatica, caritologia, soteriologia, storia dei dogmi. E-mail: lech.wolowski@upjp2.edu.pl. 
risaliamo alla sua più famosa e, nello stesso tempo, più grande opera teologica, la Trilogia ${ }^{2}$ della quale ci concentriamo sulla parte seconda, cioè la TeoDrammatica ${ }^{3}$. Nel caso di Tischner, prendiamo in considerazione soprattutto le opere che appartengono al campo della riflessione drammatica: Filozofia dramatu (La filosofia del dramma) e Spór o istnienie człowieka (L'alterco sull'esistenza dell'uomo) ${ }^{4}$.

Il concetto di persona sta senz' altro al centro di ogni riflessione drammatica. Il dramma si svolge sempre e solo tra le persone. Giustamente afferma, a proposito, Balthasar: «si riconosce qui l'immanenza del drammatico già nel concetto di persona $»^{5}$. Lo stesso concetto occupava il posto centrale anche nella riflessione tischneriana. Il suo famoso approccio agatologico-antropologico lo ha portato a formulare una nuova e assai originale definizione della persona, in quanto «agatologia incarnata» ${ }^{6}$.

Lo scopo dell'articolo è quello di analizzare, approfondire e confrontare le due posizioni dei su citati ricercatori sul concetto di persona e di individuare il loro originale contributo alla comprensione di questa nozione che, da sempre, ha avuto una posizione distinta nella storia della riflessione teologica e rimane, tuttora, al centro dell'interesse da parte della ricerca teologica contemporanea.

Per raggiungere il nostro obiettivo, abbiamo diviso il lavoro in tre parti essenziali. La prima, sarà dedicata alla comprensione balthasariana del concetto di persona; la seconda, concernerà la riflessione tischneriana sul tema; nella terza parte, ci occuperemo del confronto delle due

2 Pubblicata negli anni 1961-1987 e composta di tre parti: Herrlichkeit (Gloria), Theodramatik (TeoDrammatica), Theologik (TeoLogica).

3 Nel presente lavoro, ci riferiamo sempre all'edizione italiana TeoDrammatica, I-V, Milano 1980-1986 (trad. di G. Sommavilla).

4 Le due opere, pubblicate negli anni 1990-1998, costituiscono due parti di una grande opera tischneriana contenente la sua riflessione drammatica. A causa di mancanza delle traduzioni italiane di maggioranza delle opere tischneriane citate in questo lavoro, siamo costretti a fornire le traduzioni proprie indicate nel modo seguente: «Tr. pr.». Dove la traduzione è particolarmente difficile o dove c'è un rischio di ambiguità, forniamo anche la citazione nella lingua originale.

5 H. U. von Balthasar, TeoDrammatica III, Milano 1983, p. 216-217.

6 J. Tischner, Spór o istnienie człowieka, Kraków 2011, p. 257 [tr. pr.]. 
posizioni. La ricerca si concluderà con la conclusione e con la proposta di una prospettiva di ulteriore sviluppo.

\section{Il concetto di persona nel pensiero balthasariano}

Balthasar, nelle sue riflessioni teodrammatiche, parte dalle premesse teatrologiche e risale alla tradizione dell'antico teatro greco. Il teologo svizzero condivide con Hegel l'opinione che attribuisce al dramma una posizione eccezionale tra tutta l'attività artistica umana e che può essere ridotta alla seguente, breve affermazione: «dramma come vertice dell'arte» ${ }^{7}$. L'idea fondamentale di Balthasar è quella di «rendere le categorie drammatiche utili alla teologia cristiana» ${ }^{8}$. Una delle prime categorie teatrologiche, applicate con grande successo nel contesto teologico, è proprio quella di person $a^{9}$. Balthasar suggerisce, però, che non basta tirare fuori dal contesto teatrologico un concetto isolato, proprio come quello di persona, per applicarlo, giustamente, nel nuovo contesto teologico. Il concetto di cui parliamo, nell'ambito teatrologico, è strettamente connesso con gli altri concetti essenziali, come quelli di ruolo, di azione e di dialogo. Di conseguenza, tutte le suddette categorie devono possedere anche il loro significato teologico, e solo in tale, più ampio contesto, il concetto di persona può essere giustamente applicato e correttamente inteso nell'ambito teologico $\mathrm{o}$, più precisamente, in quello teodrammatico.

Balthasar parte, dunque, dal concetto dell'azione teodrammatica e focalizza la sua attenzione sul problema del soggetto che compie quest'azione. Da qui, viene la sua comprensione della persona in quanto «centro soggettivo dell'azione» ${ }^{10}$. L'azione compiuta dalla persona nel teodramma è strettamente legata al suo ruolo, cioè alla missione svolta da questa

H. U. von Balthasar, TeoDrammatica I, Milano 1980, p. 54.

H. U. von Balthasar, TeoDrammatica I, op. cit., p. 27.

9 Non entriamo qui nelle considerazioni storiche. Per uno sguardo storico-dogmatico sull'uso del concetto di persona nell'ambito teologico, si veda B. Sesboüé, J. Woliński, Storia dei Dogmi I. Il Dio della salvezza, Casale Monferrato 1996, p. 183nn.

10 H. U. von Balthasar, TeoDrammatica II, Milano 1982, p. 21. 
persona. Per Balthasar, i due concetti persona-missione diventeranno inseparabili fino alla loro identificazione che conduce alla nuova, originale definizione balthasariana del concetto. Nelle prossime due sezioni, esamineremo questo approccio in modo sistematico.

\subsection{Il soggetto spirituale e la persona teologica}

Balthasar, fin dall'inizio della sua riflessione teodrammatica sul concetto di persona, afferma che il problema sarà trattato da lui dal punto di vista «puramente teologico» ${ }^{11}$. Ciò è richiesto dalle controversie cristologiche del Terzo, Quarto e Quinto secolo ${ }^{12}$, a proposito della distinzione tra i concetti di natura e persona ${ }^{13}$. Balthasar dichiara apertamente che la sua intenzione è quella di non richiamarsi alla tradizione filosofica, poiché qui si tratta di un problema:

per la soluzione del quale si cerca inutilmente aiuto in campo filosofico, anche se poi nell'era cristiana e postcristiana il concetto di persona si è emancipato dal campo della teologia e corre ormai superficialmente su tutte le bocche ${ }^{14}$.

Gli riferimenti eventuali ai tentativi filosofici avranno, per Balthasar, solo lo scopo di mostrare il loro fallimento:

11 Cf. H. U. von Balthasar, TeoDrammatica III, op. cit., p. 190.

12 Balthasar è ovviamente consapevole del fatto che la storia del concetto risale a molto prima e appartiene anche al campo filosofico. Egli stesso afferma che il concetto di persona «era già in Cicerone come semplice definizione dell'individuo umano» (H. U. von Balthasar, TeoDrammatica III, op. cit., p. 190). Dal punto di vista teodrammatico, però, dove si tratta dei concetti congiunti di persona-ruolo o meglio persona-missione, la risposta fornita dal cristianesimo è, per Balthasar, assolutamente decisiva: «Persona-ruolo è un concetto limite nella dialettica tra immanenza e trascendenza, natura e sovranatura; un concetto che ha, come si dimostrerà, già occupato centralmente anche l'antichità (e tutte le culture e religioni orientali) e trova la direzione della risposta nel cristianesimo [...]» (H. U. von Balthasar, Teo Drammatica I, op. cit., p. 121).

13 Per quanto riguarda la distinzione tra questi concetti (o, nell'ambito greco, tra hypostasis e ousia) si veda H. U. von Balthasar, TeoDrammatica III, op. cit., p. 196-206; B. Sesboüé, J. Woliński, Storia dei Dogmi..., op. cit., p. 63-307.356-376; H. Pietras, Początki sporów o Trójce Święta, in: Trójca Święta. Tertulian, Przeciw Prakseaszowi, Hipolit, Przeciw Noetosowi, Kraków 1996, p. 5-28.

14 H. U. von Balthasar, TeoDrammatica III, op. cit., p. 190. 
si tratterà anzitutto di ricondurre il concetto di persona [...] là donde è venuto e di esaminare in breve le spiegazioni che, fin dal principio e fino all'età moderna, sono state tentate con il sussidio dei concetti filosofici. Il loro fallimento ci indicava la giusta strada ${ }^{15}$.

Dal punto di vista di Balthasar, «la giusta strada» sarà quella dell'approccio cristologico-teodrammatico, cioè quella della descrizione della persona del teodramma nei termini della sua in clus i o ne nella persona di Cristo ${ }^{16}$.

Nel suo cammino verso la comprensione di tale concetto, Balthasar parte dalla nozione di sogget to spirituale inteso in relazione alla nozione di nat u ra u ma na. La relazione, in modo analogico, corrisponde al rapporto tra i concetti di in di viduo e di s pe ci e. La base di questa analogia consiste nella seguente osservazione:

Tutti gli esseri viventi (almeno dagli animali superiori fino inclusivamente all'uomo) rivelano questo strano enigma: che essi hanno parte ad una natura specifica identica in tutti gli individui, ma la posseggono sempre in un modo ogni volta irripetibile e immediato ${ }^{17}$.

L'analogia di cui qui parliamo esprime, da un lato, una particolare e paradossale bipolarità e, dall'altro, una forma di retroazione tra il concetto di individuo e quello della sua specie:

Ciò che ora sul piano dell'animale significa l'inclusione di tutti gli individui di una specie in questa specie (dove la specie non esiste se non nella forma degli individui ogni volta esclusivi), è sul piano dell'uomo analogamente l'inclusione di tutti soggetti spirituali (con r efl exi o co m plet a ossia autocoscienza) nella natura umana, la quale a sua volta esiste unicamente in individui a vicenda escludentisi ${ }^{18}$.

15 H. U. von Balthasar, TeoDrammatica III, op. cit., p. 190.

16 Cf. H. U. von Balthasar, TeoDrammatica III, op. cit., p. 191.

17 H. U. von Balthasar, TeoDrammatica III, op. cit., p. 191. Anche se Balthasar non si riferisce qui esplicitamente alle opere di Gregorio di Nissa, si vede chiaramente la coincidenza tra le sue idee e quelle contenute in Grzegorz z Nyssy, O rozróżnieniu między istotą a hipostaza, in: Grzegorz z Nyssy, Drobne pisma trynitarne, Kraków 2001, p. 69-79.

18 H. U. von Balthasar, TeoDrammatica III, op. cit., p. 191-192. 
La paradossalità di cui abbiamo parlato sopra sta nel fatto che, da un lato, i concetti di individuo e di specie appaiono opporsi a vicenda ${ }^{19}$, ma, dall'altro, l'uno non può esistere e non può essere definito senza l'altro. Sembra, dunque, che entriamo in un cerchio chiuso: per definire l'individuo dobbiamo sapere la sua specie, ma per definire la specie, si deve conoscere chi sono gli individui che la formano.

Questa paradossalità, inerente al concetto di persona, costringe Balthasar a porre una domanda fondamentale: come può un soggetto spirituale sapere «chi esso è?». In altre parole: come quel soggetto si distingue non solo quantitativamente ma qualitativamente da tutti gli altri soggetti spirituali ${ }^{20}$ ?

\subsection{La persona teologica e la sua missione «In Cristo»}

Cercando la risposta alla domanda sopra menzionata, Balthasar esclude (come abbiamo già accennato) i tentativi puramente «orizzontali», cioè filosofico-intramondani. Per assicurare il chi (e non solo il che) del soggetto spirituale, non possono essere sufficienti né gli approcci oggettivi-empirici né soltanto quelli soggettivi-interpersonali ${ }^{21}$. Per uscire dal cerchio chiuso della paradossalità, si deve uscire dal piano orizzontale, per potersi riferire al so gg e t to a s soluto, cioè a Dio ${ }^{22}$. Pertanto il fondamento su cui un soggetto spirituale può autocomprendersi come per s on a (caratterizzata dal suo unico chi) si trova soltanto in Dio:

Là dove Dio dice a un soggetto spirituale chi egli sia per Lui, il Dio vero ed eternamente sussistente, dove, insieme, Dio gli dice per che cosa esiste (e gli conferisce in tal modo la sua missione su credenziale divina), là si può finalmente dire di un soggetto spirituale che è persona ${ }^{23}$.

19 Nel senso dell'opposizione tra particolarità e generalità.

20 Cf. H. U. von Balthasar, TeoDrammatica III, op. cit., p. 192.

${ }_{21}$ A proposito, Balthasar precisa che l'assicurazione del chi del soggetto spirituale «non può aver luogo a partire dalla sfera del mondo impersonale (che procura i contrassegni empirici) oppure dalla sfera dell'interpersonalità umana (dove uno non può offrire all'altro che garanzie precarie e discutibili)» (H. U. von Balthasar, TeoDrammatica III, op. cit., p. 194).

${ }_{22}$ Cf. H. U. von Balthasar, TeoDrammatica III, op. cit., p. 194.

${ }^{23}$ H. U. von Balthasar, TeoDrammatica III, op. cit., p. 194. 
Quest'affermazione può essere classificata come il primo approccio balthasariano alla definizione del concetto di persona. Balthasar non si ferma qui, ovviamente, e prosegue a concretizzare la descrizione, riferendosi all'archetipo cristologico della "definizione" di persona, nella persona del Figlio di Dio:

Questo è avvenuto una prima volta, archetipicamente, in Gesù Cristo, a cui fu conferita la sua eterna "definizione" («Tu sei il mio Figlio diletto») quando, come abbiamo visto, gli venne imprepensabilmente affidata la sua missione unica e universale e in tal modo il sapere nitidissimo di chi egli è non solo per Dio, ma fin dal principio presso Dio $(\mathrm{Gv} 1,1)^{24}$.

La pienezza del concetto di persona è raggiunta, dunque, archetipicamente e unicamente nella persona di Gesù Cristo, precisamente perché, solo in lui, la sua persona si identifica pienamente con la sua missione:

Anzitutto c'è nell'identità tra la persona di Gesù e la sua missione la pienezza infinita di ciò che è un "personaggio" drammatico: una forma (Gestalt) che nell'esecuzione del suo ruolo o perviene al suo vero volto o (nel dramma analitico) svela il suo volto nascosto. La verità della vita reale corrispondente al processo teatrale, l'identificazione della persona che senza residuo riesce in forza della sua missione, la quale pure senza residuo viene compiuta, si raggiunge nel caso di Gesù Cristo, ragion per cui egli è nel teodramma non solo il protagonista, ma l'agonista archetipico per tutti gli altri agonisti, colui dal quale essi ricevono la loro persona-identità ${ }^{25}$.

La definizione balthasariana del concetto di persona si realizza, adesso, in due passi. Il primo, che possiamo chiamare teatrologico, concerne l'identificazione della persona e la sua missione. Il secondo, crist olog i co, consiste nell'inclusione di questa missione nell'unica missione di Cristo $^{26}$. In tal modo, arriviamo alla forma finale della definizione balthasariana della persona umana:

24 H. U. von Balthasar, TeoDrammatica III, op. cit., p. 194.

25 H. U. von Balthasar, TeoDrammatica III, op. cit., p. 188-189. Vale la pena notare l'uso della parola volt o in Balthasar che non fa nessun riferimento a Lévinas e, probabilmente, non vuole avere qualunque cosa a che fare con la sua famosa teoria del volto (cf. E. Lévinas, Totalité et Infini, p. 173nn). Come vedremo nelle sezioni succedenti, in Tischner la situazione è totalmente diversa, egli, parlando del volto, quasi sempre si riferisce esplicitamente alle riflessioni di Lévinas sul tema.

26 La giustificazione dell'uso di questi due termini, cioè te atrologico e cristolog i co viene dalla terminologia e dal modo in cui Balthasar descrive il processo: «il ruolo 
In $\mathrm{Cr}$ i st o sta per ogni uomo la speranza di non rimanere un puro soggetto spirituale individuo, bensì di diventare, a partire da Dio, una persona, con un compito definito allo stesso modo in $\mathrm{Cr}$ is to $\mathrm{o}^{27}$.

Non si parla qui, ovviamente, di una definizione nel senso formale, ma di un tentativo di cogliere l'essenza teodrammatica del concetto. Un soggetto spirituale può essere chiamato persona, nella misura in cui è incluso in Cristo. L'affidamento della missione al soggetto e l'inclusione di questa missione nella missione universale di Cristo rende il soggetto una vera e propria persona. Balthasar parla, a proposito, del processo di personalizzazione del soggetto spirituale:

In quanto questa [la missione di Cristo] si estende alla sfera di tutti i soggetti spirituali (umani) e questi in tal modo si ritrovano nello "spazio" della unica persona dell'Uomo-Dio, essi vengono "personalizzati» in senso teologico, ciò vuol dire non solo negativamente "redenti", ma positivamente dotati di missioni ("carismi"), che li rendono - all'interno della missione radicale di Gesù - persone qualitativamente determinate, naturalmente in dipendenza e partecipazione verso la missione onnicomprensiva primordiale, che unicamente è identica con l'io inviato ${ }^{28}$.

Per concludere la descrizione della definizione teodrammatica del concetto di persona, proposta da Balthasar, dobbiamo notare che, tranne i due aspetti sopra menzionati (teatrologico e cristologico), essa possiede anche un terzo aspetto essenziale, quello dialogico-relazionale che richiede dal soggetto spirituale di non essere, in quanto persona, una monade chiusa in se stessa (come nel caso della classica definizione di Boezio), ma un membro della comunità aperto alla cooperazione

rappresentato da Gesù Cristo è il principio della distribuzione dei ruoli per tutti gli altri attori del dramma in quanto essi realmente hanno assegnata una parte teodrammaticamente rilevante e non recitano semplicemente un ruolo intramondano effimero su un teatro chiuso. La missione di Cristo, l'abbiamo detto più volte, è nella sua archetipicità - in quanto identica con il personaggio - il luogo da cui possono essere trasmessi ruoli o missioni (come carismi) personalizzanti ai soggetti spirituali umani» (H. U. von Balthasar, TeoDrammatica III, op. cit., p. 241).

27 H. U. von Balthasar, TeoDrammatica III, op. cit., p. 206. Si può porre la domanda: perché in Cristo? Balthasar risponde a questa domanda in modo indiretto nell'ultimo volume della sua opera: «perché la creatura deve definitivamente vivere non davanti a Dio ma in Dio» (H. U. von Balthasar, TeoDrammatica V, Milano 1986, p. 363).

${ }_{28}$ H. U. von Balthasar, TeoDrammatica III, op. cit., p. 216. 
e co-partecipazione all'universale missione di Cristo, insieme con gli altri attori del teodramma. Balthasar parla, in proposito, del processo della deprivatizzazione ossia della socializzazione:

Un soggetto spirituale umano, diventando persona per una chiamata e missione inconfondibile, viene al tempo stesso deprivatizzato, socializzato e trasformato in uno spazio e sostrato di comunità. Le persone teologiche non sono delimitate l'una rispetto all'altra come monadi allo stesso modo che i soggetti spirituali naturali ${ }^{29}$.

L'aspetto dialogico diventerà cruciale anche nella riflessione tischneriana sul concetto di persona, alla quale dedicheremo la nostra analisi nella prossima sezione.

\section{L'approccio tischneriano al concetto di persona}

Tischner inizia la sua riflessione sul concetto dal principio agatologico, cioè dalla sua famosa affermazione del primato del be ne rispetto all'ente:

La logica del bene e la logica dell'ente sono logiche diverse. Per far luce sul buio del dramma umano, occorre la logica del bene. Solo essa può giustificare l'esistenza degli enti concreti, e non viceversa. E solo essa può dare una visione dell'avventura degli uomini, ai quali non manca niente, e tuttavia, continuano a cercare un'altra terra $^{30}$.

Direttamente su questo principio, nella logica del bene, è costruita la famosa definizione tischneriana della persona come «agatologia incarnata $»^{31}$. Nelle sezioni seguenti, esamineremo come Tischner sia pervenito a questa specifica interpretazione del termine.

\footnotetext{
29 H. U. von Balthasar, TeoDrammatica III, op. cit., p. 252.

30 J. Tischner, Filozofia dramatu, Kraków 2012, p. 42 [tr. pr.].

31 J. Tischner, Spór o istnienie człowieka, op. cit., p. 257 [tr. pr.].
} 


\subsection{L'aspetto dialogico}

Accostandosi al concetto di persona nell'ambito drammatico, Tischner si chiede: «Come deve essere concepita la persona, per poter essere il partecipante del dramma?» ${ }^{32}$. Cercando una risposta, egli parte da un classico presupposto protologico, confermato dal Concilio Vaticano II, che, in modo unico, distingue l'uomo tra tutte le creature del mondo: «l'uomo [...] in terra è la sola creatura che Iddio abbia voluto per se stesso ${ }^{33}$. Tischner affermerà che l'uomo può essere chiamato l'unico e s s ere-per-sé en $^{34}$. Ma questa distinzione serve soltanto come primo passo verso la definizione della persona. Subito dopo, Tischner compie un altro passo essenziale, cioè aggiunge la dimensione dialogica:

L'essere-per-sé diventa se stesso attraverso un altro essere-per-sé. Io sono per-sé attraverso te. E tu sei per-sé attraverso me. Questo "attraverso" crea le tensioni, giacché può significare: "grazie a", "a dispetto di", "contro di" ${ }^{35}$.

La presenza della relazione dialogica e delle tensioni rende questo approccio veramente drammatico. Il principio dialogico si rivela chiaramente nell'affermazione che un soggetto non può diventare persona, se non "attraverso" un altro. Analizzando il significato del fattore "attraverso", Tischner distingue due categorie maggiori, dipendenti dal carattere della relazione dialogica in questione. Nel caso positivo, ("grazie a") la persona si costituisce secondo il principio: «Io sono me stesso grazie a te» ${ }^{36}$. Nel caso opposto ("a dispetto di", "contro di"), la relazione si costruisce nel modo negativo: «Io non sono me stesso per colpa tua» ${ }^{37}$.

Per Tischner, dunque, essere-per-sé non significa essere solo per se stesso, ma anche, o piuttosto, essere per gli altri:

32 J. Tischner, Spór o istnienie człowieka, op. cit., p. 255 [tr. pr.].

33 Costituzione Pastorale sulla Chiesa nel mondo contemporaneo, Gaudium et spes, 24.

${ }^{34}$ Cf. cit. orig.: «byt-dla-siebie», J. Tischner, Spór o istnienie człowieka, op. cit., p. 255 [tr. pr.].

35 J. Tischner, Spór o istnienie człowieka, op. cit., p. 255 [tr. pr.].

36 J. Tischner, Filozofia człowieka dla duszpasterzy i artystów, Kraków 1991, p. 12.27 [tr. pr.].

37 J. Tischner, Filozofia człowieka dla duszpasterzy i artystów, op. cit., p. 12.63 [tr. pr.]. 
La logica della genesi costitutiva dell'essere-per-sé risiede nella prospettiva che apre alla riflessione la parola "per". Io sono per te, per me, per voi... Tu sei per me, per te, per noi... Poiché siamo "per", possiamo essere, in un senso particolare - altro di oggettivo - anche accanto a sé, con sé, sopra sé, sotto sé, contro sés8.

Così «l'insieme dei sistemi temporali e spaziali, determinati dai significati della parola "per", costituisce la trama drammatica della nostra reciprocità»" ${ }^{39}$ e, allo stesso modo, la parola "per" aggiunge, alla definizione drammatica della persona, la dimensione della reciprocità: «siamo le persone, ossia i soggetti del dramma della reciprocità» ${ }^{40}$.

Identificando i punti nodali e le dimensioni principali del concetto, Tischner non cede all'illusione di arrivare ad una completa e onnicomprensiva definizione della persona, bensì preferisce parlare di mistero: «Non abbiamo la possibilità di penetrare direttamente nella profondità del mistero della persona. Possiamo soltanto indovinarlo, guardando dentro il dramma, che è il suo specchio» ${ }^{41}$.

\subsection{L'aspetto agatologico}

Dall'aspetto dialogico, Tischner scende adesso più in profondità nel mistero della persona drammatica e arriva ad un nuovo aspetto, collegato direttamente al problema della grazia:

Penetrando nella profondità del dramma della grazia e supponendo che esso è un riflesso del mistero della persona, dobbiamo chiederci: che cosa costituisce, nella persona, la condizione della possibilità di ricevere la grazia? In altre parole: grazie a che cosa, la persona è in grado di "trasformare" il male nel bene ${ }^{42}$ ?

38 Cit. orig.: «Logika genezy konstytutywnej bytu-dla-siebie kryje się w perspektywie, jaką otwiera przed myśleniem słówko “dla”. Jestem dla ciebie, dla siebie, dla was... Jesteś dla mnie, dla siebie, dla nas... Ponieważ jesteśmy “dla”, możemy być w szczególnym - innym niż rzeczowy - sensie obok siebie, ze sobą, nad sobą i pod sobą, przeciwko sobie», J. Tischner, Spór o istnienie człowieka, op. cit., p. 255 [tr. pr.].

39 J. Tischner, Spór o istnienie człowieka, op. cit., p. 255 [tr. pr.].

40 J. Tischner, Spór o istnienie człowieka, op. cit., p. 256 [tr. pr.].

${ }_{41}$ J. Tischner, Spór o istnienie człowieka, op. cit., p. 256 [tr. pr.].

${ }^{42}$ J. Tischner, Spór o istnienie człowieka, op. cit., p. 256 [tr. pr.]. 
Già dalla domanda stessa, possiamo trarre due nuove caratteristiche tischneriane della nozione di persona. Prima di tutto, la persona deve essere in grado di ricevere la grazia e, di conseguenza, deve anche essere capace di trasformare il male nel bene. Siamo, dunque, arrivati all'aspetto agatologico. Direttamente da questa osservazione, Tischner trae una delle sue più interessanti, concise, ma, nello stesso tempo, anche più enigmatiche descrizioni del concetto di persona, in quanto «agatologia incarnata» ${ }^{43}$.

La presenza del termine incarnazione indica che abbiamo qui a che fare con realtà invisibili e immateriali (grazia e bene) che entrano nella specifica relazione con quelle mondane e materiali (attraverso l'esistenza concreta, corporale dell'uomo nel mondo):

La legge dell'ente stabilisce che non appena qualcosa comincia ad esistere, da questo momento é sempre in lotta per la sopravvivenza; la legge del bene ci dice, invece, che la bontà è pronta a «morire per ciò che è Invisibile». Il luogo in cui si svolge il conflitto tra le due leggi è l'uomo: la persona che contemporaneamente «lotta per la sopravvivenza»e «fa sacrificio della sua esistenza»"

Il confronto tra corporalità e immaterialità, qui evidente, si trasferisce nel confronto tra la logica dell'ente (ontologia) e la logica del bene (agatologia). Vista da questa prospettiva, la persona è un soggetto in movimento dal piano ontologico al piano agatologico e, nello stesso tempo, dall'egoismo dell'essere solo per se stesso all'altruismo dell'essere-per-altro e, finalmente, al sacrificio-per-altro:

Il cammino del dramma corre dal desiderio - desiderio dell'essere-per-altro - al sacrificio per-altro. [...] Solo sul piano del Bene siamo uno-per-altro e uno-al posto dell'-altro ${ }^{45}$.

${ }^{43}$ J. Tischner, Spór o istnienie człowieka, op. cit., p. 257 [tr. pr.].

${ }_{44}$ Cit. orig.: «Prawo bytu na tym polega, że co raz zaistniało, to walczy o przetrwanie; prawo dobroci zaś polega na tym, że jest gotowa “umrzeć za to, co Niewidzialne”. Miejscem, na którym rozgrywa się konflikt obu tych praw, jest człowiek - osoba, która jednocześnie “walczy o byt" i “poświęca swój byt”», J. Tischner, Spór o istnienie człowieka, op. cit., p. 298 [tr. pr.].

${ }_{45}$ Cit. orig.: «Droga dramatu biegnie od pragnienia - pragnienia bycia-dla-innego do ofiary za-innego [...] Dopiero na poziomie Dobra jesteśmy jeden-dla-drugiego, jeden-za-drugiego, jeden-zamiast-drugiego», J. Tischner, Spór o istnienie człowieka, op. cit., p. 299 [tr. pr.]. 
A questo punto, la riflessione tischneriana chiaramente oltrepassa la dimensione puramente filosofico-orizzontale per raggiungere l'ambito teologico.

\subsection{L'aspetto teologico}

Nell'ultima espressione tischneriana, citata nella sezione precedente, si abbozza la dimensione teologica della sua definizione della persona. La parola «Bene», scritta qua in maiuscolo, suggerisce che trattiamo già della dimensione divina, ma, per assicurarsi che non si parla, qui, solo di un "dio dei filosofi", dobbiamo approfondire ancora la ricerca.

Tischner afferma chiaramente che senza l'elezione da parte di Dio, che è il Bene, e senza la designazione («segnazione») da Lui fatta (che nella terminologia balthasariana corrisponderebbe all'affidamento della missione) non si può parlare di vero sacrificio di vera persona ${ }^{46}$. È davvero sorprendente come la riflessione tischneriana sull'agatologia della persona comincia ad avvicinarsi, via via, al corrispondente pensiero balthasariano. Ricordiamo che, per Balthasar, l'apice della realizzazione teodrammatica della persona consisteva nell'identificazione di essa con la sua missione in Cristo. Qui, con espressioni come «segnazione», «unzione», «sacrificio della sua esistenza», «morire per ciò, che è Invisibile», Tischner si avvicina ad una simile comprensione. La situazione diventa ancora più interessante quando ci rendiamo conto che Tischner non toc$\mathrm{ca}$, qui, l'aspetto teologico soltanto nel suo insieme, ma, come Balthasar (usando, però, una diversa terminologia) scopre la dimensione cristologica del concetto di persona. Infatti, anche per Tischner, alla fine, tutta la dignità e tutto il valore della persona umana viene dalla persona del Figlio di Dio:

Dio il Creatore ha creato all'inizio il mondo. Ma il semplice riferimento alla esistenza del mondo non significa che questo mondo e gli uomini che vivono in esso hanno qualche valore. $O$ che un ente creato vale più di un altro ente. Solo l'elezione

46 Tischner infatti afferma: «Colui, che è stato "eletto" dal Bene, era anche "segnato" verso qualcosa, o meglio verso qualcuno. "Segnazione" significa "imprimere il segno" - il segno della scelta [...]. "Segnazione" è anche "unzione" - la consacrazione per il ministero e per il sacrificio» (J. Tischner, Spór o istnienie człowieka, op. cit., p. 298 [tr. pr.]). 
e la morte del Figlio di Dio sulla croce è stata la vera creazione dei valori. Il Figlio dell'Uomo, che ha fatto il sacrificio per l'uomo, ha creato l'uomo come un valore. Il sacrificio assoluto ha stabilito il valore assoluto della persona umana ${ }^{47}$.

Parlando del sacrificio nel contesto della dignità e del valore che rappresenta in sé la persona umana, non si può prescindere dalla profonda metafora del volto che Tischner prende in prestito da Lévinas, ma che poi, in seguito, sviluppa nella sua riflessione originale ${ }^{48}$.

Il volto, sia per Lévinas che per Tischner, costituisce, da un lato, l'elemento che distingue la persona dalle altre nozioni e realtà e, dall'altro lato, rappresenta il punto d'incrocio tra immanenza e trascendenza in questo mondo:

La parola chiave che descrive l'incontro è il volto. Le cose hanno le apparenze, le persone hanno i volti. Le cose appaiono attraverso le apparenze, i volti si rivelano. I volti sono le tracce della Trascendenza. Verso che cosa ci portano queste tracce? Quali drammi aprono davanti a noi ${ }^{49}$ ?

Per Tischner, il volto rivela la dimensione agatologica della persona nel senso pieno e costituisce l'illustrazione della definizione tischneriana della persona, in quanto «incarnazione dell'agatologia». La persona si rivela e si definisce attraverso il suo volto, che incarna, simbolizza e rivela, nello stesso tempo, tutta la bontà (o cattiveria) presente (o nascosta) nella persona stessa. Il bene e il male, rivelati nell'incontro e nel dialogo attraverso i volti delle persone, definiscono i rapporti tra loro e il carattere del dramma che essi sperimentano:

Il volto si rivela come un dono dell'orizzonte agatologico: l'orizzonte, in cui il bene e il male assumono la forma del dramma, mentre il dramma preannunzia la possibilità di tragedia o di vittoria dell'uomo. Nel dramma, il bene e il male non sono lontani uno dall'altro come nel regno dei concetti, ma si intrecciano nella contemporaneità, nello spazio comune, nello stesso uomo ${ }^{50}$.

47 J. Tischner, Nadzieja czeka na słowo, Kraków 2011, p. 263 [tr. pr.].

48 Vale la pena notare che, in questo momento, perdiamo la corrispondenza diretta tra il pensiero di Tischner e quello di Balthasar ed entriamo nel campo delle soluzioni caratteristiche da Tischner, appoggiate sulle idee di Lévinas.

${ }^{49}$ J. Tischner, Filozofia dramatu, op. cit., p. 27 [tr. pr.].

50 J. Tischner, Filozofia dramatu, op. cit., p. 82 [tr. pr.]. 
Inoltre, sul volto resta sempre una traccia, la traccia della trascendenza che rivela la dimensione teologica della persona. Infatti, sul volto si trovano tracce diverse. Tutto il dramma umano è, in certo senso, impresso sul volto dell'uomo, ma Tischner intende indicare, qui, quella traccia più fondamentale, lasciata, sul volto della creatura, dal Creatore stesso, la traccia del Bene assoluto, «la traccia della traccia»:

Che cos'è il volto dell'altro? È la traccia lasciataci da Dio, e dunque il segno che siamo stati scelti. Questa è la traccia che non si può abbandonare per correre, continuamente, dietro a chi viene seguito. Questa è la traccia della traccia ${ }^{51}$.

Siamo arrivati, dunque, al senso più profondo della definizione tischneriana del concetto di persona, cioè allo strato teologico sempre presente e sempre visibile proprio nel volto di ogni persona. Concludiamo, con questa osservazione, la descrizione dell'approccio tischneriano al tema, per poter effettuare, nella parte successiva, il confronto delle posizioni rappresentate dai due autori.

\section{Il confronto delle due posizioni}

Balthasar e Tischner sono pienamente consapevoli che, per sviluppare un nuovo - teodrammatico - approccio, in teologia, occorre ridefinire il concetto di persona per adattarlo alle esigenze delle nuove sfide. I loro atteggiamenti sul problema sembrano essere vicini, anche se non mancano punti di divergenza.

Entrambi gli autori sono decisivamente convinti che la teologia drammatica debbe rompere con la classica definizione di persona introdotta da Boezio e fissata, per lunghi secoli, in teologia, soprattutto nella riflessione scolastica e dominante in essa fino alla nascita del personalismo. Ambedue sono egualmente d'accordo che, anche se l'approccio personalistico (dialogico-relazionale) è definitivamente più adeguato alle considerazioni drammatiche, non basta fermarsi a questa tappa della riflessione.

51 Cit. orig.: «Czym jest twarz innego? Jest śladem pozostawionym nam przez Boga, a więc znakiem naszego wybrania. Jest to ślad, którego porzucić nie można, by biec dalej za śledzonym. Jest to ślad śladu.», J. Tischner, Filozofia dramatu, op. cit., p. 59 [tr. pr.]. 
Entrambi sentono la necessità di aggiungere qualcosa di specificamente drammatico, tratto dalla tradizione teatrologica. Questo è il loro contributo originale al tema, ma ciascuno di loro ha attuato tale contributo in modo differente, attraverso la scelta dei diversi elementi teatrologici che caratterizzano l'originalità delle loro definizioni. Tuttavia, dobbiamo aggiungere, che le loro scelte non si contraddicono, bensì, si completano a vicenda.

In Tischner, la base della definizione si colloca nell'agatologia. Di conseguenza, per lui, la persona del dramma è nient'altro che l'«agatologia incarnata» caratterizzata dal suo volto. In Balthasar, la definizione è fondata sulla cristologia e pertanto l'essenza della persona consiste nell'«inclusione in Cristo», caratterizzata dalla missione. In generale, dunque, entrambi si distaccano dalle definizioni filosofiche classiche e risalgono, nelle loro definizioni, agli elementi teatrologici incorporati rispettivamente, nell'agatologia e nella cristologia.

Il volto tischneriano si riferisce all'antico concetto teatrologico di $\pi \rho o ́ \varsigma \omega \pi$ ov (prosopon, la maschera), mentre la missione balthasariana è nient'altro che il diretto equivalente teodrammatico del ruolo affidato all'attore, nel contesto teatrale.

\subsection{L'aspetto dell'autenticità}

Le due relazioni analogiche: il volto - la maschera e la missione - il ruolo riflettono l'essenza del pensiero teatrologico-teologico di entrambi gli autori. La scelta dei termini non viene, qui, a caso. Sia il volto che la missione portano in sè un profondo sentimento di autenticità, di verità e di serietà, mentre la maschera e il ruolo ci portano subito alla mente un pensiero sulla simulazione, sul gioco e, a volte, persino sulla falsità.

Tischner, riflettendo sulla differenza tra il volto e la maschera, nota: «la maschera non è la cortina e non è il volto. La cortina soltanto nasconde il volto, la maschera mente. [...] Incontrando la maschera non possiamo non domandare sul volto. Cerchiamo il volto in qualche luogo fuori delle maschere. Cerchiamo così, come si cerca la verità ${ }^{52}$.

52 J. Tischner, Filozofia dramatu, op. cit., p. 74.79 [tr. pr.]. 
In modo simile, Balthasar sottolinea l'autenticità della missione teodrammatica che si estende fino all'identificazione tra essa e la persona alla quale è stata affidata: «la sfera della missione si identifica sempre con quella dell'io che fa tutt'uno con essa» ${ }^{53}$.

Arriviamo qui a una distinzione essenziale tra l'approccio teatrologico e quello teologico. Più precisamente, si chiarisce la profonda differenza tra il dramma teologico ed il dramma teatrale. Nella realizzazione del dramma teatrale, l'attore accetta un certo ruolo, affidato a lui dal regista, mette, poi, la maschera (il trucco, la caratterizzazione) e recita la sua parte sulla scena. Dopo la rappresentazione, l'attore abbandona il suo ruolo scenico e torna alla sua vita normale.

Invece nel dramma teologico, i protagonisti-cristiani svolgono sulla scena del mondo le missioni affidate loro da Cristo, e così «vengono personalizzati in senso teologico; ciò vuol dire [...] dotati di missioni (c a ris $\mathrm{mi}$ ), che li rendono - all'interno della missione radicale di Gesù - persone qualitativamente determinate» ${ }^{54}$, e testimoniano, con i loro volti, di fronte agli altri e di fronte a Dio, tutto ciò che costituisce il loro dramma:

grazie al volto possiamo cogliere l'essenza dell'incontro: incontrare l'altro significa incontrarlo nel suo volto [...] perciò si deve dire che l'epifania del volto sta alla fonte di ogni dramma inteso profondamente ${ }^{55}$.

\subsection{L'aspetto della libertà}

Il rapporto tra teatrologia e teologia nel contesto delle considerazioni drammatiche sulla persona, descritto da noi nella sezione precedente, nell'aspetto del problema di autenticità, non si esaurisce in questo solo aspetto. Per approfondire ancora la questione, vale la pena mettere in rilievo un altro aspetto che rivela una ulteriore essenziale differenza tra il dramma teatrale e quello teologico. Si tratta dell'aspetto della libertà delle persone che partecipano al dramma.

\footnotetext{
53 H. U. von Balthasar, TeoDrammatica III, op. cit., p. 229-230.

54 H. U. von Balthasar, TeoDrammatica III, op. cit., p. 216.

55 J. Tischner, Filozofia dramatu, op. cit., p. 80-81 [tr. pr.].
} 
Nell'ambito teatrale, non appena il ruolo viene affidato all'attore, egli è costretto a recitare la parte prescritta fino alla fine della rappresentazione. Nella critica teatrale, si parla, in verità, dell' «interpretazione» della parte, ma, almeno per quanto riguarda il teatro classico, l'interpretazione non ammette l'improvvisazione e, di conseguenza, non lascia molto spazio alla libertà dei protagonisti.

La persona del dramma teologico, almeno nell'approccio tischneriano e balthasariano, sta, invece, sempre di fronte ad una scelta libera e personale. La comprensione teologica del dramma, cioè il teodramma, consiste, precisamente, nel fatto che in ogni momento della vita cristiana, il protagonista deve scegliere tra i diversi gradi e tipi del bene (o del male) e non c'è suggeritore alcuno che ci dica che cosa fare.

Balthasar osserva, in proposito, che altrimenti il dramma teologico sarebbe ridotto ad un certo tipo di teatro delle marionette ${ }^{56}$, che contradirebbe totalmente il rapporto fondamentale della sua Teodrammatica, cioè il rapporto tra la libertà finita e la libertà infinita ${ }^{57}$.

Anche per Tischner, la libertà dei protagonisti costituisce l'aspetto fondamentale di ogni dramma tra l'uomo e Dio. C'è, inoltre, qualcosa di più: la libertà delle persone umane è possibile e deve la sua esistenza esclusivamente alla libertà primordiale immanente delle persone divine della Santissima Trinità:

L'uomo è creato a immagine e somiglianza di Dio, e Dio è la Santissima Trinità. Dio unico nella Trinità è libero? [...] Se Dio fosse davvero libero, non solo esteriormente ma anche interiormente, la genealogia della libertà umana sarebbe ad un certo grado chiarificata. Dio creando l'uomo a «sua immagine» dovrebbe dargli qualcosa dalla sua libertà ${ }^{58}$.

56 In questo tema cf. H. U. von Balthasar, TeoDrammatica I, op. cit., p. 128-178.

57 In particolare, vale la pena riportare qui la seguente affermazione di Balthasar: «La creazione della libertà finita da parte dell'infinita è il punto di partenza della teodrammatica» (H. U. von Balthasar, TeoDrammatica II, op. cit., p. 256). A proposito si veda anche F.G. Brambilla, Antropologia teologica, Brescia 2009, p. 106-111.

${ }_{58}$ J. Tischner, Spór o istnienie człowieka, op. cit., p. 378 [tr. pr.]. Per essere precisi, dobbiamo acconsentire che, nella citazione sopra riportata, Tischner afferma soltanto la dipendenza della libertà umana su quella divina. Per quanto riguarda la sua opinione sulla libertà interiore della Trinità stessa si veda J. Tischner, Życie wewnętrzne Boga, in: Ksiądz na manowcach, Kraków 2007, p. 88-101. 
In tal modo, arriviamo all'interessante conclusione che non solo Balthasar (nel caso di cui, la conclusione, sarebbe ovvia), ma anche Tischner fissa la libertà della persona umana all'infinita profondità della libertà (intra)trinitaria.

Conclusioni finali e prospettive per un ulteriore sviluppo

Per concludere le nostre considerazioni, vale la pena notare che Balthasar e Tischner hanno offerto, insieme, un nuovo contributo e perfino hanno introdotto una nuova apertura nella lunga storia dello sviluppo del concetto di persona, nella riflessione teologica.

È importante aggiungere che, entrambi, parlano in modo indipendente, usando una terminologia diversa, ma con una voce quasi unanime per quanto riguarda l'idea principale. L'uno mette l'accento sul rapporto missione-ruolo, l'altro, sul volto-maschera, ma il messaggio è univoco: la nozione della persona teologica può e deve essere approfondita attraverso le considerazioni delle realtà teatrologiche, trasferite in modo appropriato nel campo teologico.

Possiamo dunque affermare che, grazie all'originale approccio drammatico al concetto di persona, introdotto da entrambi gli autori, non soltanto è stata arricchita la comprenssione teologica del concetto, ma è stata anche notevolmente approfondita la relazione tra teologia e teatrologia. Secondo il nostro parere, il contributo, qui presentato, non è ancora abbastanza riconosciuto ed apprezzato sia nella teologia sia nella teatrologia. Il problema merita, dunque, un serio e profondo studio ulteriore. 


\section{Sommario}

L'approccio agatologico-cristologico al concetto di persona nella riflessione teatrologica-teologica di Hans Urs von Balthasar e di Józef Tischner

In quest'articolo, presentiamo l'approccio contemporaneo alla comprensione teologica del concetto di persona, formatosi nella corrente del pensiero teodrammatico, rappresentato da H. U. von Balthasar e da J. Tischner. Descriviamo gli aspetti fondamentali antropologici (agatologici-dialogici) e teologici (cristologicitrinitari) caratteristici degli approcci di entrambi gli autori. Prestiamo attenzione particolare ai contributi originali di Balthasar e di Tischner al problema preso in esame, soprattutto per quanto riguarda l'uso della terminologia teatrologica nelle considerazioni riguardanti la descrizione teologica del concetto di persona. L'asse dell' analisi la costituisce il paragone tra due teatrologiche-teologiche analogie chiave, riguardante la nozione di persona introdotte dagli autori su menzionati e concernente, rispettivamente, il rapporto r uolo-mis si o ne (Balthasar) e quello di maschera-volto (Tischner).

Parole chiave: persona, teodramma, ruolo-missione, maschera-volto, Balthasar,

Tischner

The agathological-christological approach to the concept of person in the theatrological-theological thought of Hans Urs von Balthasar and Józef Tischner

In this article we present the contemporary approach to the theological understanding of the concept of person according to the theodramatical current of thought represented by H. U. von Balthasar and J. Tischner. We describe the basic anthropological (agathological-dialogical) and teological (christologicaltrinitological) aspects characteristic for the approach of both authors. We pay a particular attention to the original contribution of Balthasar and Tischner in the analyzed problem, especially as far as the application of theatrological terminology to the considerations regarding the theological notion of person is concerned. The axis of the analysis is the comparison of the two key theatrological-theological analogies regarding the notion of person introduced by the above mentioned authors concerning respectively the relation role-mission (Balthasar) and mask-face (Tischner).

Keywords: person, theodrama, role-mission, mask-face, Balthasar, Tischner 


\section{Bibliografia}

Balthasar H. U. von, Herrlichkeit. Eine theologische Ästhetik, I-III, Einsiedeln 19611969 (traduzione italiana di G. Sommavilla in sette volumi: Gloria. Un'estetica teologica, I-VII, Milano 1985-1991).

Balthasar H. U. von, Theodramatik, I-IV, Einsiedeln 1973-1983 (traduzione italiana di G. Sommavilla in cinque volumi:TeoDrammatica, I-V, Milano 1980-1986).

Balthasar H. U. von, Theologik, I-III, Einsiedeln 1985-1987 (traduzione italiana di G. Sommavilla in tre volumi: TeoLogica, I-III, Milano 1989-1992).

Brambilla F. G., Antropologia teologica. Chi è l'uomo perché te ne curi?, Brescia 2009.

Grzegorz z Nyssy, o rozróżnieniu między istota a hipostaza, in: Grzegorz z Nyssy, Drobne pisma trynitarne, Kraków 2001, p. 69-79.

Lévinas E., Totalité et Infini. Essai sur l'extériorité, La Haye 1986.

Pietras H., Początki sporów o Trójcę Święta, in: Trójca Święta. Tertulian, Przeciw Prakseaszowi, Hipolit, Przeciw Noetosowi, Kraków 1996, p. 5-28.

Sesboüé B., Wolinski J., Storia dei Dogmi I. Il Dio della salvezza, Casale Monferrato 1996.

Tischner J., Filozofia człowieka dla duszpasterzy i artystów, Kraków 1991.

Tischner J., Filozofia dramatu, Kraków 2012.

Tischner J., Nadzieja czeka na słowo. Rekolekcje, Kraków 2011.

Tischner J., Podgladanie Pana Boga, “Znak” 511 (1997), p. 8-24.

Tischner J., Spór o istnienie człowieka, Kraków 2011.

Tischner J., Życie wewnętrzne Boga, in: J. Tischner, Ksiądz na manowcach, Kraków 2007, p. 88-101. 